\title{
Robust Control Allocation among Overactuated Spacecraft Thrusters under Ellipsoidal Uncertainty
}

\author{
Chenyang Duan, Shijie Zhang, Yafei Zhao, and Xianren Kong \\ Research Center of Satellite Technology, Harbin Institute of Technology, Harbin 150080, China \\ Correspondence should be addressed to Shijie Zhang; sjzhang@hit.edu.cn
}

Received 31 March 2014; Revised 24 May 2014; Accepted 24 May 2014; Published 19 June 2014

Academic Editor: Hamid Reza Karimi

Copyright ( 2014 Chenyang Duan et al. This is an open access article distributed under the Creative Commons Attribution License, which permits unrestricted use, distribution, and reproduction in any medium, provided the original work is properly cited.

\begin{abstract}
Spacecrafts with overactuated and redundant thrusters can work normally once some of them are out of work, which improves the reliability of spacecraft in orbit. In this way, the desired command of controller needs to be dynamically allocated among thrusters. Considering that uncertain factors may appear in forms of dynamics, installation errors, thrust deviations, or failures, this paper proposes a robust control allocation under ellipsoidal uncertainty. This method uses the uncertainty ellipsoid set to describe the uncertainty of the thrusters firstly and establish the thrust allocation robust reference model and then transforms it into a cone optimization model which can be solved as an optimized problem. Finally, this paper adopts the interior-point method for solving the optimization problem. In this way, difficulties of solving the problem caused by parameter uncertainties are avoided effectively. Finally, we take satellite rendezvous and docking as simulation scenarios; it is verified that the cumulative distribution error and maximum error can be reduced by more than $15 \%$ when the random error of control efficiency matrix is $5 \%-20 \%$; also, precision of thruster allocation is improved.
\end{abstract}

\section{Introduction}

Thrusters are used widely in spacecraft control. In order to ensure the reliability of spacecraft orbiting, redundancy configuration is often adopted in the existing design concept to increase the redundancy of system, which can make its nonuniqueness of the expected control command assigning schemes given by control algorithm. Traditionally, the distribution schedule is commonly formulated in advance according to the layout of thrusters. In this way, problems can be solved effectively and have advantages of distribution under certain uncertainties. However, this method takes up a lot of onboard storage space and is hard to allocate in real time according to the satellite status. Therefore, it is necessary to give a thrust allocation algorithm, which can not only save the onboard resources but also improve the orbit precision of spacecraft. Modern distribution schemes usually bring kinds of optimization ideas, which make the thruster output in conformity with the control instruction as far as possible [1]. For example, Bemporad et al. [2] and Tøndel et al. [3] put forward multiple parametric quadratic programming problems, which describes the thruster allocation problem as a piecewise linear function containing a target parameter; Durham gives a direct allocation method, solving the thruster allocation problem in the form of a polyhedron $[4,5]$ and so on.

In the process of practical work, the thruster installation location, installation angle, and space environment disturbances all can lead to the changes of thruster distribution, and thruster forces and torques acting on the satellite are deviated from the expected value. If these uncertainties are ignored, obvious force or torque distribution deviation will happen; therefore, it is necessary to research robust thruster allocation algorithm under thruster uncertainties.

When dealing with uncertainty problem, the existing thruster allocation algorithm generally based on the premise of actuators can accurately output hypothesis to improve the robustness of the system through introducing adaptive control $[6,7]$. However, the installation error of the actuator is not considered in this algorithm, and the error often has large influence on control system. In another method, the influence of thruster error is considered, and errors are estimated online by introducing the adaptive update when making the thrust allocation [8]. Some works develop a robust control 


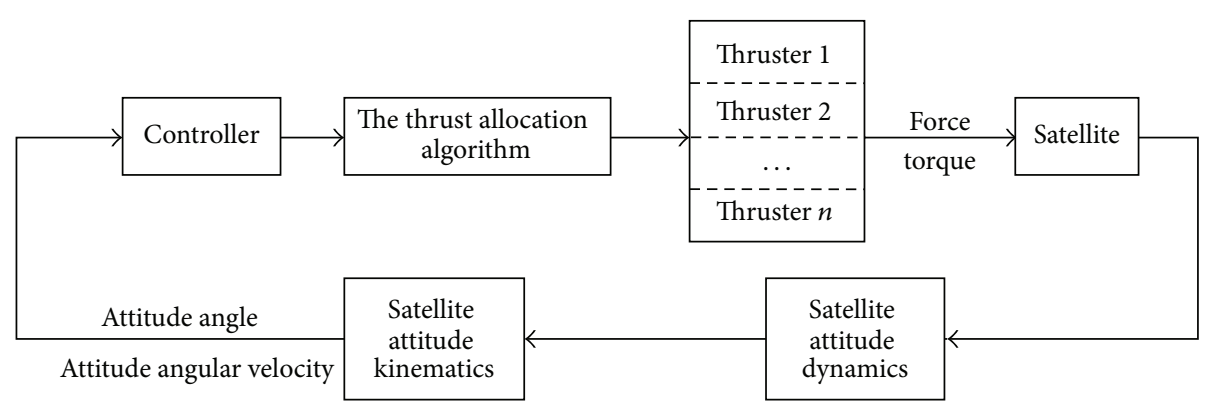

FIGURE 1: The principle diagram of thrust allocation.

allocation scheme for rigid spacecraft attitude stabilization in the presence of actuator partial loss fault, actuator failure, and actuator misalignment. These methods are observer-based [9] or detection-based estimations [10]. Therefore, the force or torque states in each moment need to be measured in these methods, and the quantity of these states is not easy to get in the practical application of satellite, which greatly limits the application of this method. Some papers take parameter uncertainty, output tracking, disturbance attenuation, and fuel cost into consideration in the controller level. They introduce a new Lyapunov approach, so the controller design problem can be transferred into a convex optimization problem subject to linear matrix inequality (LMI) constraints [11]. Wan et al. proposes an observer-based robust guaranteed cost control method for thrust-limited rendezvous in nearcircular orbits [12]. There are also some scholars proposing a dynamic control allocation strategy based on the theory of robust predictive control, in view of the actuator output polyhedron uncertainty problems $[13,14]$.

However, these methods above do not model for uncertainty, or the models need to be descripted more accurately when dealing with the thruster uncertainty problem. A method solving this problem from thruster allocation level is lacking.

Uncertainty ellipsoid set method is a method of probability distribution, which describes the uncertainty set to a determinate parameters model by restraining the uncertainty boundary. When modeling the deviations such as thruster installation deviation in this method, the boundary point information is the only need. Even when the endpoint information is unknown, it is can be designed according to the experience. So this method is widely used in the uncertainty description of the actuator of missile, aircraft, and satellite. By introducing uncertainty sets in the thruster allocation problem, the uncertainty distribution problems can be equivalent to a determinacy distribution model, which can be solved using a mathematical optimization algorithm. The uncertainty ellipsoid set provides an effective way for thruster allocation uncertainty problem, because it only needs the uncertainty boundary information and is easy to build.

The method proposed in this paper is to solve the control allocation problems under control matrix uncertainties in the control allocation level, rather than control law level. In this way, control law does not need to change or switch in order to adjust for uncertainties and the entire control schedule can be simplified.

In this paper, the thrust allocation problem with uncertainty controls effectiveness matrix is studied (Figure 1). First, we analyze the controls effectiveness matrix deviation caused by the factors such as thruster installation and take uncertainty ellipsoid set to express this control efficiency matrix uncertainty. Then we transform the thruster allocation model into a model which can be solved in math based on the idea of transforming the uncertain parameters optimization problem into certain parameters optimization problem and solve it by the interior-point method. Finally, we validate the feasibility of the algorithm.

\section{Problem Description}

Thrust allocation problem is a control technology of dynamically allocating the expected control force among the redundancy configuration thrusters to achieve the goal of optimal allocation and improve the control performance of the system. The core of this problem is a control allocation problem. This paper first gives the mathematical model of the thrust allocation and then preliminarily describes the uncertainty of the controls effectiveness matrix in this model. Finally, it gives a preliminary model of thrust allocation problem considering the uncertainty.

The characteristics of the thruster is that the thrust it produced can only be in one direction, so the attitude control torque can only be produced in one direction, too. If we need the attitude control torque in all directions, multiple thrusters should be combined. There are $n$ thrusters installed on the spacecraft. In the body fixed coordinate system $O x_{b} y_{b} z_{b}$, assuming that the principal axes are coinciding with the three inertial principal axes of satellite, the position vector of the $i$ th thruster relative to satellite centroid is $\mathbf{d}_{i}=\left(x_{i}, y_{i}, z_{i}\right) \mathbf{e}=\mathbf{d}_{i}^{T} \mathbf{e}$, where $x_{i}, y_{i}$, and $z_{i}$ are the components of thrusters in three axes, respectively, $\mathbf{e}=\left[\mathbf{e}_{x}, \mathbf{e}_{y}, \mathbf{e}_{z}\right]^{T}$ express the three basis vectors of body coordinate system, and the unit thrust vector matrix produced by the $i$ th thruster is $\mathbf{e}_{i}=\left(\cos \alpha_{i} \cos \beta_{i}\right.$, $\left.\cos \alpha_{i} \sin \beta_{i}, \sin \alpha_{i}\right) \mathbf{e}$, see Figure 2 .

Assuming a single thruster can provide continuous thrust, for fixed thruster, it can be equivalent by means 


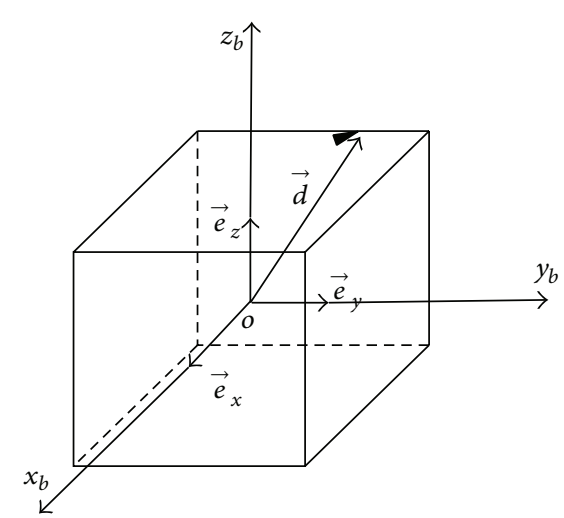

(a)

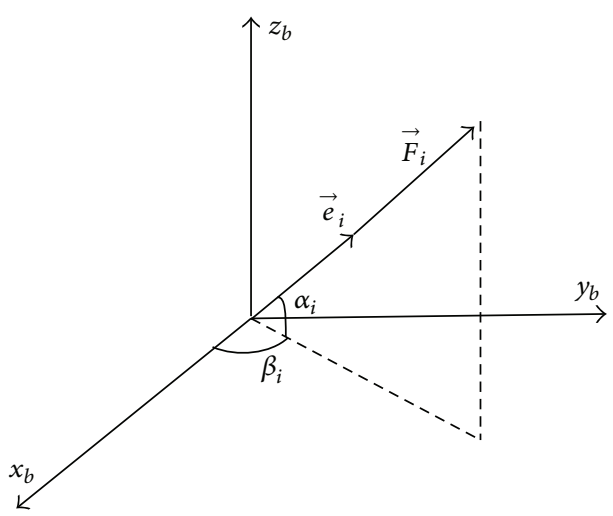

(b)

FIGURE 2: The layout diagram of the $i$ th thruster.

of pulse width modulation. The thrust vector of the $i$ th thruster is

$$
\mathbf{F}_{i}=F_{i} \mathbf{e}_{i}
$$

where

$$
0 \leq F_{i} \leq F_{i \max } .
$$

$F_{i \text { max }}$ is the maximum thrust which can be produced if the thruster is switched on during an on/off cycle.

The resultant force of all the thrusts produced by thrusters acting on the satellite is

$$
\mathbf{U}=\sum_{i=1}^{n} \mathbf{F}_{i}=\sum_{i=1}^{n} F_{i} \mathbf{e}_{i} .
$$

Let the number of thrusters be $n$; define the thrust vector of thruster as

$$
\mathbf{F}=\left[F_{1}, F_{2}, \ldots, F_{n}\right]^{T} .
$$

The resultant force of thrust can be written as

$$
\mathbf{U}=\mathbf{B F},
$$

where

$$
\mathbf{B}=\left[\mathbf{e}_{1}, \ldots, \mathbf{e}_{n}\right]=\left[\mathbf{b}_{1}, \ldots, \mathbf{b}_{n}\right] .
$$

Considering the torque $\mathbf{T}$ acting on the satellite generated from the thrust, the torque produced by one thruster is

$$
\mathbf{T}_{i}=\mathbf{d}_{i} \times \mathbf{F}_{i}=\left(\mathbf{d}_{i} \times \mathbf{e}_{i}\right) F_{i} .
$$

So the resultant torque acting on the satellite is

$$
\mathbf{T}=\sum_{i=1}^{n} \mathbf{T}_{i}=\sum_{i=1}^{n}\left(\mathbf{d}_{i} \times \mathbf{e}_{i}\right) F_{i},
$$

which is written as

$$
\mathbf{T}=\mathbf{A F},
$$

where

$$
\mathbf{A}=\left[\mathbf{d}_{1} \times \mathbf{e}_{1}, \ldots, \mathbf{d}_{i} \times \mathbf{e}_{i}, \ldots, \mathbf{d}_{n} \times \mathbf{e}_{n}\right]=\left[\mathbf{a}_{1}, \ldots, \mathbf{a}_{n}\right] .
$$

Noticing that the elements in $\mathbf{A}$ and $\mathbf{B}$ have the relation of

$$
\mathbf{a}_{i}^{T} \mathbf{b}_{i}=0 .
$$

Then, the thruster control allocation model gets

$$
\begin{array}{ll}
\text { opt. } & f(\mathbf{F}) \\
\text { s.t. } & {\left[\begin{array}{l}
\mathbf{U} \\
\mathbf{T}
\end{array}\right]=\left[\begin{array}{l}
\mathbf{B} \\
\mathbf{A}
\end{array}\right] \mathbf{F}} \\
& 0 \leq \mathbf{F} \leq \mathbf{F}_{\max } .
\end{array}
$$

If we only consider the attitude control, the model could be written as

$$
\begin{array}{ll}
\text { opt. } & f(\mathbf{F}) \\
\text { s.t. } & \mathbf{T}=\mathbf{A F} \\
& 0 \leq \mathbf{F} \leq \mathbf{F}_{\max } .
\end{array}
$$

Defining $\mathbf{A}$ as control efficiency matrix and seeing from the derivation process of the model, the matrix $\mathbf{A}$ is closely related to the thruster position and angle. In the actual process of satellite on-orbit operation, there are many factors leading to the uncertainty of the controls effectiveness matrix, such as the Sun's gravity and Earth's gravity gradient on the impact of satellite center of mass, and the installation angle and position errors appeared in the process of installing the thrusters. Defining $\mathbf{A}$ as nominal control efficiency matrix, $\Delta \mathbf{A}(t)$ as deviation of the controls effectiveness matrix, and $\mathbf{A}_{\text {act }}$ as actual control efficiency matrix, we have

$$
\mathbf{A}_{\mathrm{act}}=\Delta \mathbf{A}+\mathbf{A} .
$$

The actual torque acting on the satellite is

$$
\mathbf{T}_{\mathrm{act}}=\mathbf{A}_{\mathrm{act}} \mathbf{F} \text {. }
$$


The deviation of thrust distribution is

$$
\mathbf{T}_{\mathrm{act}}-\mathbf{T}=\mathbf{A}_{\mathrm{act}} \mathbf{F}-\mathbf{A F}=\Delta \mathbf{A F} .
$$

For solving the uncertainty problem of control efficiency matrix, there is no difference between the model of attitudeorbit control and the model of attitude control. Therefore, for simplification, the derivations below are all based on the model of attitude control.

Taking minimum error as index, the model of control effectiveness uncertainty matrix of satellite attitude control can be written as

$$
\begin{array}{ll}
\min & \|\mathbf{F}\| \\
\text { s.t. } & \mathbf{T}=\mathbf{A}_{\mathrm{act}} \mathbf{F} \\
& 0 \leq \mathbf{F} \leq \mathbf{F}_{\max } .
\end{array}
$$

Noticing the uncertain parameters in this model makes it difficult to be solved. Without considering the condition of model uncertainty, pseudoinverse method is generally used [15].

\section{Robust Control Allocation Algorithm}

For the uncertainty problem of control efficiency matrix in the process of satellite thruster allocation, a robust control allocation algorithm is given in this section. This method models the uncertain parameters in the model and then transforms the uncertain parameters model into a thruster allocation model, which can be solved in mathematical method. The specific process is as follows:

(1) describe the uncertainty of the control effectiveness matrix using uncertainty ellipsoid set;

(2) model the deviation of control efficiency matrix as uncertainty ellipsoid is set to get thruster allocation models with deterministic parameters;

(3) transform the thruster allocation model reasonably; in order to solve it, use mathematical method;

(4) give the "satisfied solution" of model by adopting the mathematical method, so as to solve the thrust allocation problem.

3.1. Description of the Control Effectiveness Matrix Uncertainty Using Uncertainty Ellipsoid Set. First, we give the definition of ellipsoid and uncertainty ellipsoidal set.

Definition 1 (the definition of ellipsoid). Assuming the $\mathbb{R}^{k}$ dimensional Euclidean geometry space, there are matrix $\mathbf{A} \in$ $\mathbb{R}^{m \times n}$ and vector $\mathbf{x}=\left[x_{1}, \ldots, x_{n}\right]^{T}$, where $m \leq k$ and $n \leq k$. Then, we call the set which satisfies the equation

$$
U=\left\{f(\mathbf{x}) \in \mathbb{R}^{k} \mid\|\mathbf{A} \mathbf{x}\|_{2} \leq 1\right\}
$$

as the ellipsoid determined by matrix $\mathbf{A}$ and function $f(\cdot)$, where $f(\mathbf{x})$ is the projection function of $\mathbf{x}^{n} \rightarrow \mathbf{x}^{k}$. There are three kinds of situations specifically as follows:
(1) $m=n=k$, and the matrix $\mathbf{A}$ is nonsingular; in this situation, the ellipsoid determined by matrix $\mathbf{A}$ is a normal ellipsoid in $\mathbb{R}^{k}$-dimensional space;

(2) $m=n<k$, and the matrix $\mathbf{A}$ is nonsingular; in this situation, the ellipsoid determined by matrix $\mathbf{A}$ is in $\mathbb{R}^{k}$-dimensional space; the vector $\mathbf{x}$ has no definition in $(k-n)$-dimensional space, so it can be imaged that the projection of set $U$ is a "flat" ellipsoid in the $\mathbb{R}^{k}$ dimensional space;

(3) $m \neq n$, and the matrix $\mathbf{A}$ is singular; in this situation, the vector $\mathbf{x}$ is radially unbounded in $(n-m)$ dimensional space, the projection of set $U$ is an "elliptical cylinder" in the $\mathbb{R}^{k}$-dimensional space.

Definition 2 (the definition of uncertainty ellipsoidal set). If the following conditions are satisfied, we call uncertainty set $\Xi$ as uncertainty ellipsoid set:

(1) $\Xi$ is the intersection of limited multiple ellipsoid;

(2) $\Xi$ is bounded;

(3) there are matrix $\mathbf{A}$ and function $f(\cdot)$, which makes $\Xi=\left\{f(\mathbf{x}) \in \mathbb{R}^{k} \mid\|\mathbf{A} \mathbf{x}\|_{2} \leq 1\right\}$.

The advantages of taking uncertainty ellipsoid set model as a quantitative description of uncertainty are as follows:

(1) from the view of mathematics, the ellipsoid has simple parametric expressions and is easy to implement;

(2) for every random uncertainty problem with probability distribution, a suitable uncertainty ellipsoid set could be found to describe it;

(3) the intersection of uncertainty ellipsoid set can approximate more complex sets of uncertainty;

(4) the optimization model with uncertain set is easier to be analyzed and more convenient for computer processing.

As mentioned above, thruster allocation problem is to give a reasonable solution of the allocation problem satisfying the thruster limit constraints. So the core of the proposed robust optimization method is solving the robust reference model for the parameter combinations in each of the uncertain sets; by satisfying all the constraint conditions, the "appropriate" solution is obtained. That is to say, we pay more attention to the performance under the state of large works, rather than the performance for once.

3.2. The Robust Model Reference. The robust optimization method for solving the problems of controls effectiveness matrix uncertainty, whose core idea is to transform the original control allocation model into a convex optimization problem which can be solved by mathematical method. The key of this problem is to establish the corresponding alternative model, called robust reference model, and then find the optimal solution using the related theory of the optimization [16]. 
In view of the practical problems, how to find the robust reference model is the key to solve the problem of control allocation with uncertain parameters. Obviously, robust reference model is closely related to the original problem model. So we should first make sure the original problem of control allocation model determines which decision variables leads to uncertainty and then determine the range of variation of the uncertainty variables and give a full description, so as to determine the objective function, constraint function of control allocation model, and variation range of uncertain variables. Ben-Tal and Nemirovski studied uncertainty model under the linear and nonlinear model and proved that the robust reference model of the original control allocation problem could be obtained by mathematical derivation [17].

The establishment process of robust reference model is as follows:

(1) analyze the original problem and determine the objective function constraint conditions and optimization variables of the problem;

(2) determine the uncertain variables and the variation range of them;

(3) derive the equivalent model to eliminate the problem of the model which cannot be solved and brought by the uncertainty variable.

According to the control allocation model above, establish corresponding robust reference model and analyze it. Take the fuel optimal as the optimization goal, and, considering the uncertainty of the controls effectiveness matrix, the robust reference model can be written as

$$
\begin{array}{ll}
\min & \|\mathbf{F}\| \\
\text { s.t. } & \mathbf{A}_{\text {act }} \mathbf{F}=\mathbf{T}, \quad \forall \mathbf{a}_{i} \in \Xi_{i}^{(1)}, \forall i \\
& 0 \leq \mathbf{F} \leq \mathbf{F}_{\max },
\end{array}
$$

where $\mathbf{a}_{i}$ is the $i$ th column of the uncertain matrix $\mathbf{A}$ and belongs to the uncertain set $\Xi_{i}^{(1)}$.

3.3. The Cone Optimization Model for Robust Thrust Distribution. In view of the uncertainty of control efficiency matrix in the thruster allocation, make the following three assumptions:

(1) control efficiency matrix $\mathbf{A}$ is uncertain, but its variable elements $a_{i j}$ are random in the uncertainty set and change independently of each other;

(2) the random variables $a_{i j}$ have normal value, that is, the ideal value;

(3) the matrix parameters obey normal distribution; the uncertain sets is a standard ellipsoid.

Theorem 3. Robust control allocation problem with uncertainty ellipsoid is as follows:

$$
\begin{array}{ll}
\min & \|\mathbf{x}\| \\
\text { s.t. } & \mathbf{A x} \geq \mathbf{b}, \quad \forall \mathbf{a}_{i} \in \mathbf{\Xi}_{i}, \forall i \\
& 0 \leq \mathbf{x} \leq \mathbf{x}_{\text {max }} .
\end{array}
$$

If it is satisfied,

(1) matrix A has uncertainty, but the values in each column are all in the scope of ellipsoid:

$$
\Xi_{i}=\left\{\mathbf{a}_{i} \mid \mathbf{a}_{i}=\overline{\mathbf{a}}_{i}+\Theta_{i} \mathbf{k}_{i},\left\|\mathbf{k}_{i}\right\| \leq \rho\right\},
$$

where $i=1, \ldots, n, \overline{\mathbf{a}}_{i}$ is the normal value and $\Theta_{i}$ is symmetric positive definite matrix;

(2) The uncertainties of column $\mathbf{a}_{i}$ are independent of each other; the model above is equivalent to the following cone optimization problem:

$$
\begin{array}{ll}
\min & \|\mathbf{x}\| \\
\text { s.t. } & \overline{\mathbf{a}}_{i}^{T} \mathbf{x}-\rho\left\|\Theta_{i} \mathbf{x}\right\| \geq \mathbf{b}_{i}, \quad \forall i=1, \ldots, n \\
& 0 \leq \mathbf{x} \leq \mathbf{x}_{\max } .
\end{array}
$$

Proof. The robust control allocation problem with ellipsoid uncertainty can be rewritten as

$$
\begin{array}{ll}
\min & \|\mathbf{x}\| \\
\text { s.t. } & \mathbf{a}_{i}^{T} \mathbf{x}+\mathbf{x}^{T} \Theta_{i} \mathbf{k}_{i} \geq \mathbf{b}_{i} \\
& \mathbf{\Omega}=\left\{k_{i} \mid\left\|k_{i}\right\| \leq \rho\right\} \\
& 0 \leq \mathbf{x} \leq \mathbf{x}_{\max } \\
& \forall i=1, \ldots, n .
\end{array}
$$

Further, it can be rewritten into

$$
\begin{array}{ll}
\min & \|\mathbf{x}\| \\
\text { s.t. } & \mathbf{a}_{i}^{T} \mathbf{x}-\mathbf{b}_{i}+\min _{\mathbf{k}_{i} \in \Omega} \mathbf{x}^{T} \Theta_{i} \mathbf{k}_{i} \geq 0 \\
& \mathbf{\Omega}=\left\{k_{i} \mid\left\|k_{i}\right\| \leq \rho\right\} \\
& 0 \leq \mathbf{x} \leq \mathbf{x}_{\max } \\
& \forall i=1, \ldots, n .
\end{array}
$$

As $\Theta_{i}$ is positive definite and $\mathbf{x} \geq 0$, equation

$$
\min _{\mathbf{k}_{i} \in \mathbf{\Omega}} \mathbf{x}^{T} \Theta_{i} \mathbf{k}_{i}=-\rho\left\|\Theta_{i}^{T} \mathbf{x}\right\|
$$

is set up; substituting it into (24) yields

$$
\begin{array}{ll}
\min & \|\mathbf{x}\| \\
\text { s.t. } & \mathbf{a}_{i}^{T} \mathbf{x}-\mathbf{b}_{i}-\rho\left\|\Theta_{i}^{T} \mathbf{x}\right\| \leq 0 \\
& 0 \leq \mathbf{x} \leq \mathbf{x}_{\max } \\
& \forall i=1, \ldots, n .
\end{array}
$$

According to the attitude control thrust allocation model in (19), the model can be rewritten as

$$
\begin{array}{ll}
\min & \|\mathbf{F}\| \\
\text { s.t. } & {\left[\begin{array}{c}
\mathbf{A}_{\text {act }} \\
-\mathbf{A}_{\text {act }}
\end{array}\right] \mathbf{F} \geq\left[\begin{array}{c}
\mathbf{T} \\
-\mathbf{T}
\end{array}\right]} \\
& 0 \leq \mathbf{x} \leq \mathbf{x}_{\text {max }} .
\end{array}
$$


Let

$$
\mathbf{C}=\left[\begin{array}{c}
\mathbf{A}_{\text {act }} \\
-\mathbf{A}_{\text {act }}
\end{array}\right], \quad \mathbf{D}=\left[\begin{array}{c}
\mathbf{T} \\
-\mathbf{T}
\end{array}\right]
$$

thereupon

$$
\begin{array}{ll}
\min & \|\mathbf{F}\| \\
\text { s.t. } & \mathbf{C F} \geq \mathbf{D} \\
& 0 \leq \mathbf{F} \leq \mathbf{F}_{\max } .
\end{array}
$$

The model has the form of Theorem 3 and can be converted into cone quadratic optimization problem

$$
\begin{array}{ll}
\min & \|\mathbf{F}\| \\
\text { s.t. } & \rho\left\|\Theta_{i}^{T} \mathbf{F}\right\| \geq \mathbf{c}_{i}^{T} \mathbf{F}-\mathbf{d}_{i} \\
& 0 \leq \mathbf{F} \leq \mathbf{F}_{\max } \\
& \forall i=1, \ldots, 2 n .
\end{array}
$$

Through the transformation above, the robust reference model can be transformed to a quadratic cone optimization problem, and then the thruster allocation problem with uncertain parameters can be transformed into a quadratic cone optimization problem with certain parameters, and it can be solved by mathematical methods.

\subsection{The Solution Algorithm Based on Interior-Point Method.} We transform the control allocation problem with uncertain controls effectiveness matrix into a quadratic cone programming problem; considering the need of calculation efficiency, the numerical method based on interior-point method can be used; the interior-point method can refer to reference [18]. Here is the simple idea, and the corresponding solving process is given.

In this paper, the European norm is 2-norm by default; if we write the inequality constraints in model (30) as

$$
\rho\left\|\Theta_{i}^{T} \mathbf{F}(t)\right\| \leq \mathbf{c}_{i}^{T} \mathbf{F}(t-\Delta T)-\mathbf{d}_{i},
$$

where $\Delta T$ is the sampling period, so the robust allocation problem of thrust is actually the following mathematical problem:

$$
\begin{array}{r}
\min \|\mathbf{A x}-\mathbf{b}\| \\
\|\mathbf{x}\| \leq \rho_{0} .
\end{array}
$$

The dual problem of it is

$$
\begin{gathered}
\|\mathbf{A} \mathbf{x}-\mathbf{b}\| \leq \mu, \\
\|\mathbf{x}\|=\min .
\end{gathered}
$$

Theorem 4. $\mathbf{x}$ is the solution of (31), if and only if $\lambda>0$; let

$$
\begin{gathered}
\mathbf{A}^{T}(\mathbf{A} \mathbf{x}-\mathbf{b})=-\lambda \mathbf{x} \\
\varphi=\mathbf{x}^{T} \mathbf{x}-\rho_{0}^{2}=0 .
\end{gathered}
$$

The solving process adopts cross iteration method for (34a) and (34b), that is, solving $\mathbf{x}$ for (34a) according to the given $\lambda$, and then adjusts the value of $\lambda$ according to the sign of $\varphi$ until the obtained $\lambda_{0}$ and $\mathbf{x}_{0}$ make (33) an equation. The key of this method is to simplify $\mathbf{A}$ to a double diagonal matrix by Householder transformation before solving.

Let $\mathbf{e}_{1}=(1,0, \ldots, 0)^{T}$, for any vector $\mathbf{a}=\left(\alpha_{1}, \ldots, \alpha_{n}\right)^{T} \in$ $\mathbb{R}^{n}$; there is the Householder transformation

$$
\mathbf{V}=\mathbf{I}-\frac{\mathbf{u u}^{T}}{\pi}, \quad \pi=\frac{1}{2} \mathbf{u}^{T} \mathbf{u},
$$

making $\mathbf{V a}=-\sigma \mathbf{e}_{1}$, where $\mathbf{u}=\mathbf{a}+\sigma \mathbf{e}_{1}$ and $\sigma=\|\mathbf{a}\| \operatorname{sign}\left(\alpha_{1}\right)$, and if $\mathbf{a}=0$, there is $\mathbf{V}=\mathbf{I}$.

Make

$$
\mathbf{A}=\left[\mathbf{a}_{1}, \mathbf{a}_{2}, \ldots, \mathbf{a}_{n}\right] .
$$

Let $\mathbf{Q}_{1}$ be the Householder transformation taking $\mathbf{a}_{1}$ in place of $\mathbf{a}$; then we have

$$
\mathbf{B}_{1}=\mathbf{Q}_{1} \mathbf{A}=\left[\begin{array}{cccc}
\delta_{1} & \beta_{12} & \cdots & \beta_{1 n} \\
0 & \beta_{22} & \cdots & \beta_{2 n} \\
\vdots & \vdots & & \vdots \\
0 & \beta_{m 1} & \cdots & \beta_{m n}
\end{array}\right],
$$

where $\delta_{1}=\left\|\mathbf{a}_{1}\right\| \operatorname{sign}\left(\alpha_{11}\right)$; let $\mathbf{b}_{1}=\left(\beta_{12}, \ldots, \beta_{1 n}\right)^{T}$; then

$$
\mathbf{U}^{\prime}=\mathbf{I}_{n-1}-\frac{\mathbf{u}^{\prime} \mathbf{u}^{\prime T}}{\pi^{\prime}}, \quad \pi^{\prime}=\frac{1}{2} \mathbf{u}^{\prime T} \mathbf{u}^{\prime},
$$

where $\mathbf{u}^{\prime}=\mathbf{b}_{1}+\left\|\mathbf{b}_{1}\right\|\left(\operatorname{sign} \beta_{12}\right) \mathbf{e}_{1}$; it is obvious that

$$
\mathbf{b}_{1}^{T} \mathbf{U}^{\prime}=\left(\varepsilon_{1}, 0, \ldots, 0\right) \text {. }
$$

While $\varepsilon_{1}=\left\|\mathbf{b}_{1}\right\| \operatorname{sign} \beta_{12}$, thus let

$$
\mathbf{U}_{1}=\left(\begin{array}{cc}
1 & 0 \\
0 & \mathbf{U}^{\prime}
\end{array}\right)
$$

there is

$$
\mathbf{A}_{1}=\mathbf{B}_{1} \mathbf{U}_{1}=\left[\begin{array}{ccccc}
\delta_{1} & \varepsilon_{1} & 0 & \cdots & 0 \\
0 & \beta_{22}^{\prime} & \beta_{23}^{\prime} & \cdots & \beta_{2 n}^{\prime} \\
0 & \beta_{32}^{\prime} & \beta_{33}^{\prime} & \cdots & \beta_{3 n}^{\prime} \\
\vdots & \vdots & \vdots & & \vdots \\
0 & \beta_{m 2}^{\prime} & \beta_{m 2}^{\prime} & \cdots & \beta_{m n}^{\prime}
\end{array}\right]
$$

It is easy to be seen that repeating the process above yields

$$
\begin{aligned}
\mathbf{A}_{1} & =\mathbf{A} \\
\mathbf{A}_{2} & =\mathbf{Q}_{1} \mathbf{A}_{1} \mathbf{U}_{1} \\
& \vdots \\
\mathbf{A}_{n-1} & =\mathbf{Q}_{n-2} \mathbf{A}_{n-2} \mathbf{U}_{n-2} \\
\mathbf{A}_{n} & =\mathbf{Q}_{n-1} \mathbf{A}_{n-1} \mathbf{U}_{n-1} \\
\mathbf{A}_{n+1} & =\mathbf{Q}_{n} \mathbf{A}_{n} .
\end{aligned}
$$


A could be transformed into double diagonal matrix $\mathbf{A}_{n+1}$, the $\mathbf{Q}_{i}, \mathbf{U}_{i}$ here are all Householder transformation.

For overactuated spacecraft, the control efficiency matrix $\mathbf{A}_{m \times n}$ satisfies $m<n$ (for underactuated spacecraft, the relationship is $m>n$ ); there are $2 m-1$ Householder transformations $\mathbf{Q}_{1}, \ldots, \mathbf{Q}_{m-1}, \mathbf{U}_{1} \ldots \mathbf{U}_{m}$; make

$$
\mathbf{Q}_{m-1} \cdots \mathbf{Q}_{1} \mathbf{A U}_{1} \cdots \mathbf{U}_{m}=(\mathrm{LO})
$$

where

$$
(\mathrm{LO})=\left[\begin{array}{ccccc}
\delta_{1} & 0 & \cdots & \cdots & 0 \\
\varepsilon_{1} & \delta_{2} & 0 & \cdots & 0 \\
0 & \varepsilon_{2} & \ddots & \ddots & \vdots \\
\vdots & \ddots & \ddots & \delta_{m-1} & 0 \\
0 & \cdots & 0 & \varepsilon_{m-1} & \delta_{m}
\end{array}\right] .
$$

By carrying out the above process, the problem to be solved could be converted into

$$
\left[\begin{array}{ccccc}
\delta_{1} & 0 & \cdots & \cdots & 0 \\
\varepsilon_{1} & \delta_{2} & 0 & \cdots & 0 \\
0 & \varepsilon_{2} & \ddots & \ddots & \vdots \\
\vdots & \ddots & \ddots & \delta_{m-1} & 0 \\
0 & \cdots & 0 & \varepsilon_{m-1} & \delta_{m}
\end{array}\right]\left[\begin{array}{c}
\xi_{1} \\
\xi_{2} \\
\xi_{3} \\
\vdots \\
\xi_{n}
\end{array}\right]=\left[\begin{array}{c}
\varphi_{1} \\
\varphi_{2} \\
\varphi_{3} \\
\vdots \\
\varphi_{n}
\end{array}\right] .
$$

Resolve $\mathbf{A}$ as $\mathbf{A}=\mathbf{L U}$, where

$$
A_{1}=\left[\begin{array}{cccccccccccccccc}
0 & 0 & 0 & 0 & 0 & 0 & 0 & 0 & -0.16 & 0.16 & 0 & 0 & 0.16 & -0.16 & 0 & 0 \\
0 & 0 & 0 & 0 & 0 & 0 & 0 & 0 & 0 & 0 & -0.16 & 0.16 & 0 & 0 & 0.16 & -0.16 \\
0.16 & -0.16 & 0.16 & -0.16 & -0.16 & 0.16 & -0.16 & 0.16 & 0 & 0 & 0 & 0 & 0 & 0 & 0 & 0
\end{array}\right] .
$$

Taking the attitude control of tracking satellite in last period of rendezvous and docking process as the background, the rotational inertia of minisatellite is $\mathbf{I}=[0.1500 ; 00.150$; $000.15] \mathrm{kg} \cdot \mathrm{m}^{2}$; the initial attitude quaternion is $\mathbf{q}\left(t_{0}\right)=$ $\left[\begin{array}{llll}0.7035 & -0.4708 & 0.3430 & 0.4073\end{array}\right]^{T}$, corresponding to the initial attitude angles $\varphi_{0}=30^{\circ}, \theta_{0}=60^{\circ}$, and $\psi_{0}=-50^{\circ}$; the initial attitude angular velocity is $\omega_{b 0}=\left[\begin{array}{lll}0 & 0 & 0\end{array}\right]^{T}$; PD control law is adopted in the controller; the parameters of control law are $k_{p}=0.32$ and $k_{d}=0.8$.

$$
\begin{aligned}
\mathbf{L} & =\left[\begin{array}{ccccc}
\beta_{1} & 0 & \cdots & & 0 \\
\varepsilon_{1} & \beta_{2} & & & \\
0 & \varepsilon_{2} & \ddots & \ddots & \vdots \\
\vdots & \ddots & \ddots & \beta_{m-1} & 0 \\
0 & \cdots & 0 & \varepsilon_{m-1} & \beta_{m}
\end{array}\right], \\
\mathbf{U} & =\left[\begin{array}{ccccc}
1 & \gamma_{1} & 0 & \cdots & 0 \\
0 & 1 & & \ddots & \vdots \\
& & \ddots & \ddots & 0 \\
\vdots & & \ddots & 1 & \gamma_{n-1} \\
0 & \cdots & & 0 & 1
\end{array}\right] .
\end{aligned}
$$

So the problem can be solved.

The whole idea of solving is as follows:

(1) giving the initial value of $\lambda_{0}$, which satisfies,

(2) writing the matrix $\mathbf{A}$ as the component form of column vector and finding the homologous corresponding transformation matrix by (35)-(40),

(3) transforming A into a double diagonal matrix by (41),

(4) making LU decomposition for A by (45), transforming it into the form of (46), and solving $\mathbf{x}$ in (34a),

(5) substituting $\mathbf{x}$ into (34b) and judging the sign of $\varphi$,

(6) changing the value of $\lambda$ and repeating the process above until $\varphi=0$ to find the solution $\mathbf{x}$.

\section{Simulation and Analysis}

The given robust control allocation algorithm is simulated in this section. The thruster configuration is as shown in the Figure 3 (numbers 1-16 are thrusters).

The control efficiency matrix of the thruster attitude control is

Figure 4 shows the curves of attitude quaternion and attitude angular velocity by adopting the robust thrust allocation strategy. It can be seen that the angular velocity of tracking satellite relative to main satellite tends to 0 , and the direction of it is adjusted; the rendezvous and docking mission can be fulfilled. It is proved that this robust thrust allocation algorithm can adjust the initial state deviation into the expectation state, which proves the feasibility of this algorithm. 


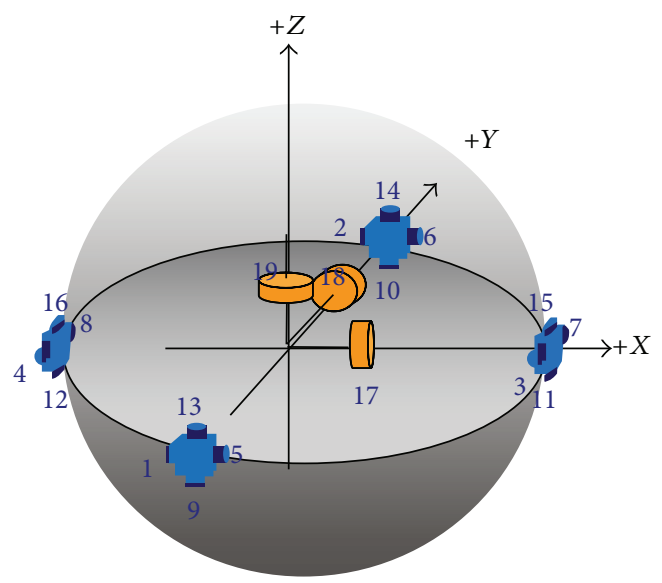

Figure 3: The Thrusters of Micro-SIM.

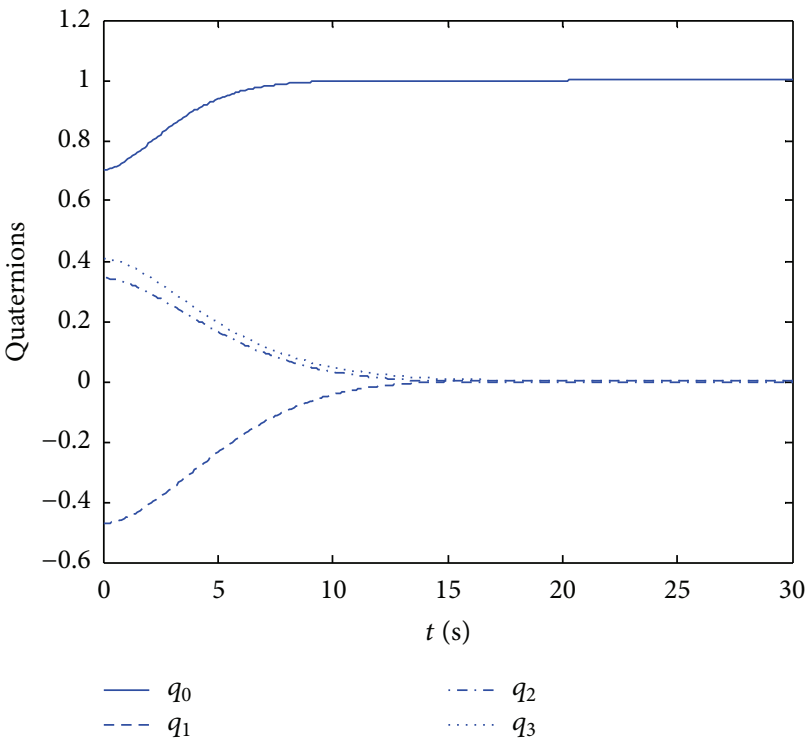

(a)

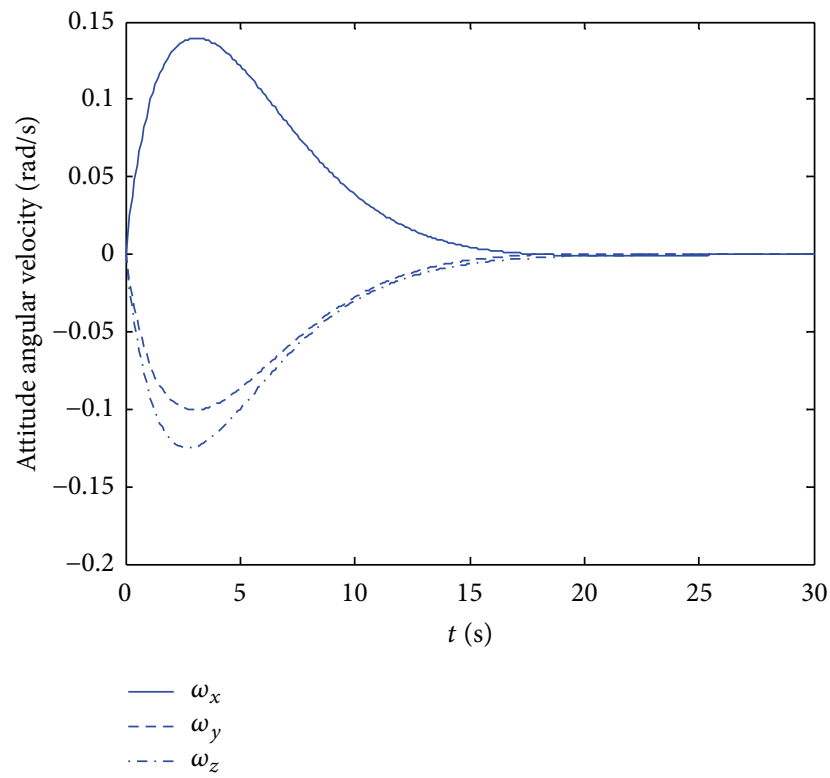

(b)

Figure 4: The curves of attitude quaternion and attitude angular velocity.

Figure 5 and Table 1 show the results of 10 random simulations when the control efficiency matrix is with the uncertainty of $20 \%$. It can be seen that the robust thrust allocation method could fulfill the mission of attitude control. The changing curves of error are smooth and steady, the cumulative deviation of torque in three axes directions decreases by about $20 \%$, and the maximum distribution deviation decreases by about $26 \%$, which proves the effectiveness of this method.

Figure 6 and Table 2 show the results of 10 random simulations when the control efficiency matrix is with the uncertainty of $10 \%$. It can be seen that the robust thrust allocation method is effective as well. The range ability in error of this robust allocation algorithm decreases and distributes more uniform in both sides of 0 -line. The result shows that the cumulative deviation of torque decreases by about $40.7 \%$, and the maximum distribution deviation decreases by about $16.3 \%$

Figure 7 and Table 3 show the results of 10 random simulations when the control efficiency matrix is with the uncertainty of $5 \%$. The result shows that the cumulative deviation of torque decreases by about $36.5 \%$, and the maximum distribution deviation decreases by about $19.9 \%$. The above simulation shows that this proposed robust allocation algorithm is correct and effective.

\section{Conclusion}

The thrust allocation problem of overactuated spacecraft is researched in this paper, and the deviation of the controls effectiveness matrix is analyzed. By the description of ellipsoid uncertainty, the uncertain model parameters problem 


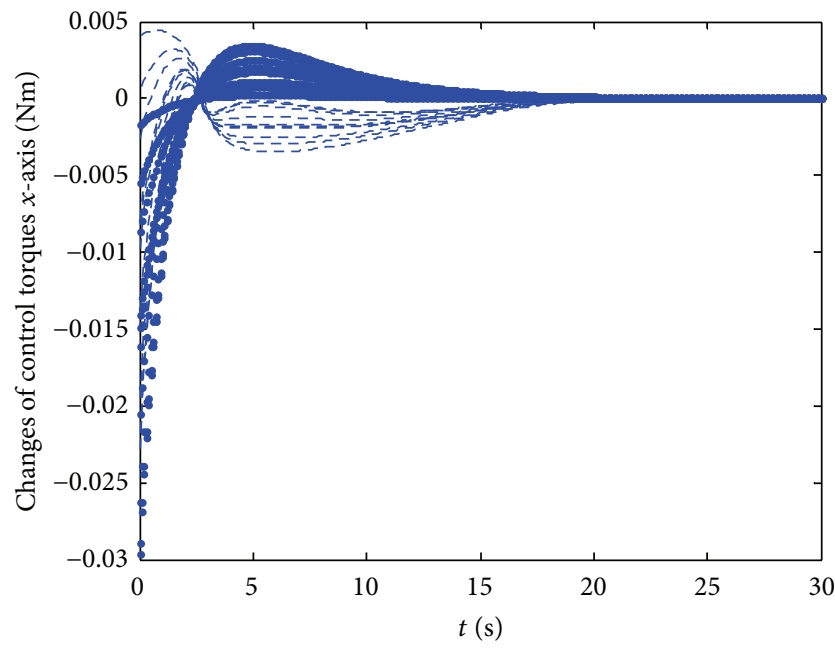

- Pseudoinverse allocation

- - Robust thruster allocation

(a)

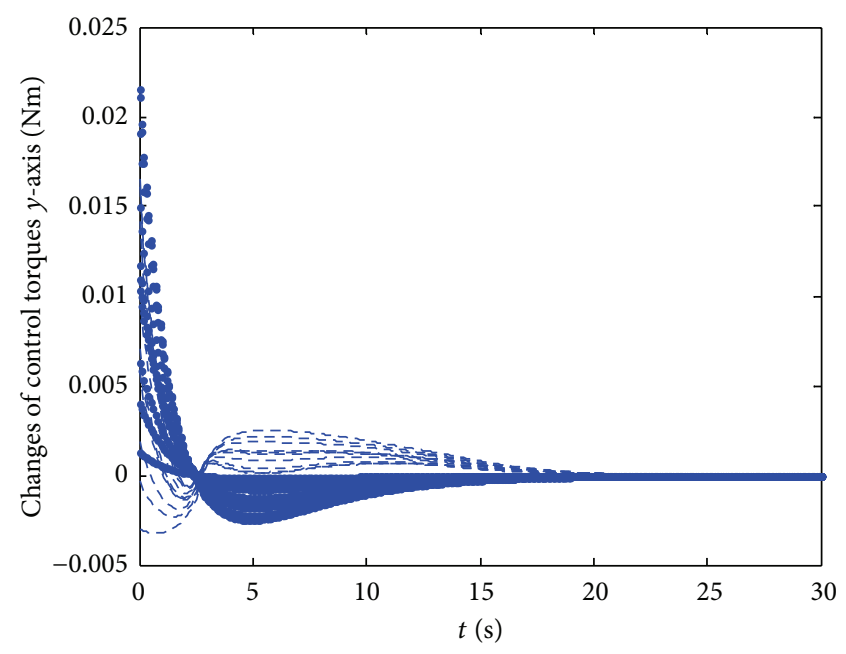

- Pseudoinverse allocation

- - Robust thruster allocation $(\mathrm{Nm})$

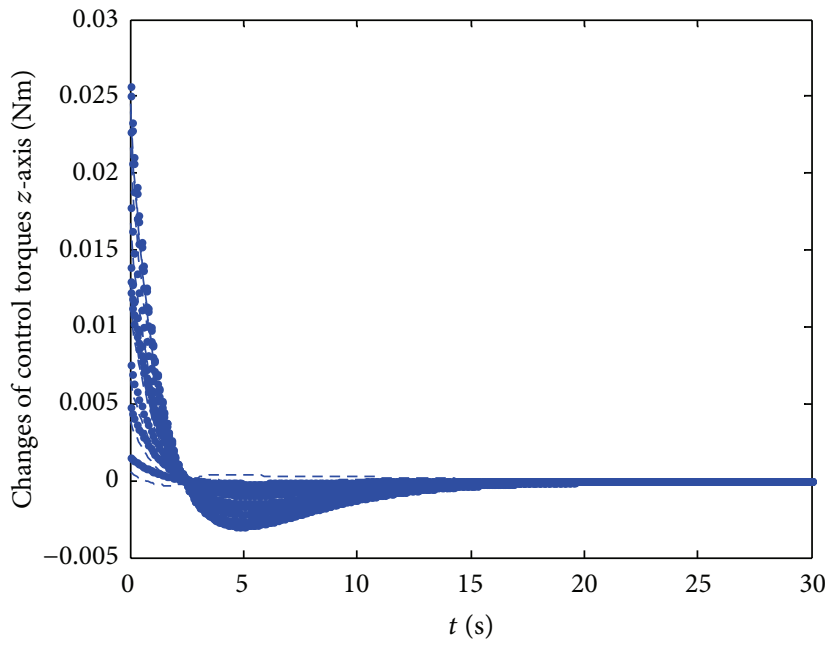

- Pseudoinverse allocation

- - - Robust thruster allocation

(c)

FIGURE 5: The curves of torque distribution deviation when the control efficiency matrix is with the uncertainty of $20 \%$.

TABLE 1: The simulation result when the control efficiency matrix is with the uncertainty of $20 \%$.

\begin{tabular}{llccccc}
\hline & \multicolumn{2}{l}{ The cumulative error of torque $(\mathrm{N} / \mathrm{m})$} & \multicolumn{2}{c}{ The maximum distribution deviation of torque $(\mathrm{N} / \mathrm{m})$} \\
& $x$-axis & $y$-axis & $z$-axis & $x$-axis & $y$-axis & $z$-axis \\
\hline Pseudoinverse thruster allocation & 0.3245 & 0.2364 & 0.2807 & 0.0181 & 0.0132 & 0.0156 \\
Robust thruster allocation & 0.2662 & 0.1936 & 0.2191 & 0.0116 & 0.0085 & 0.0146 \\
\hline
\end{tabular}




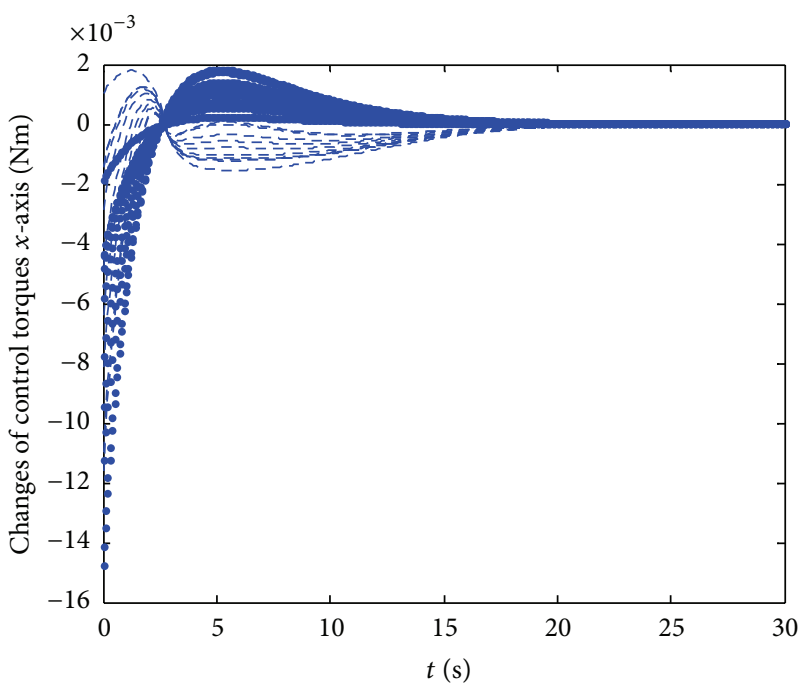

- Pseudoinverse allocation - - - Robust thruster allocation

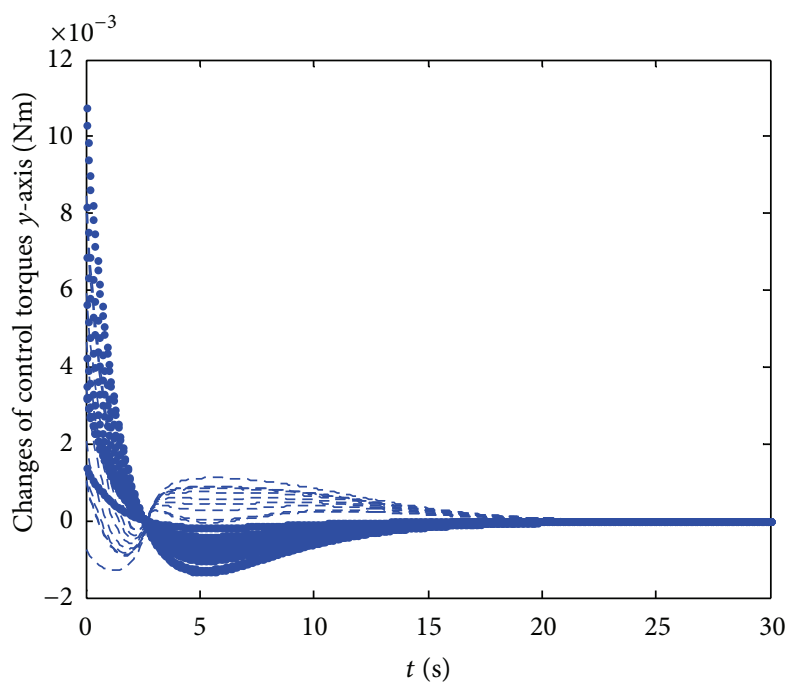

- Pseudoinverse allocation

- - Robust thruster allocation (Nm)

(a)

(b)

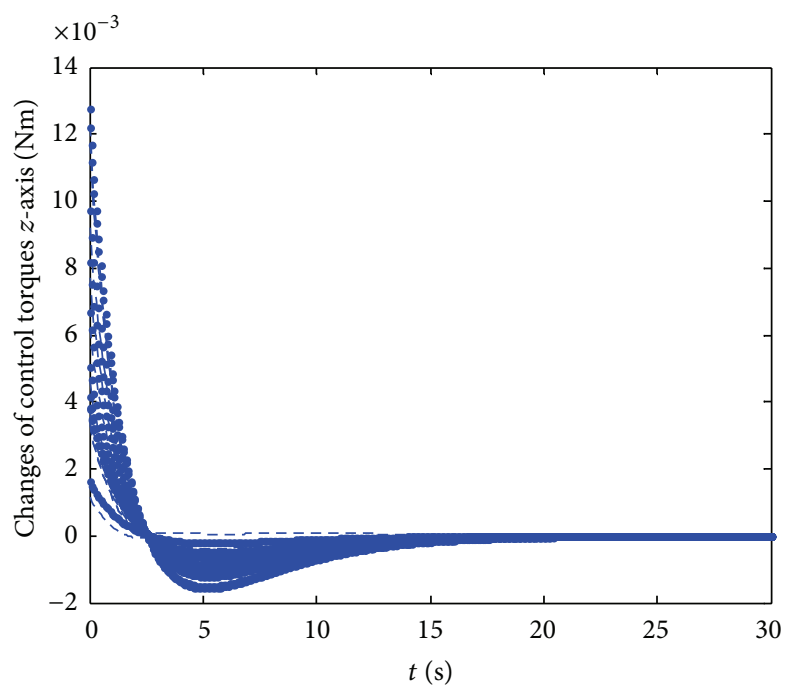

- Pseudoinverse allocation

- - Robust thruster allocation

(c)

FIGURE 6: The curves of torque distribution deviation when the control efficiency matrix is with the uncertainty of $10 \%$.

TABLE 2: The simulation result when the control efficiency matrix is with the uncertainty of $10 \%$.

\begin{tabular}{lccccccc}
\hline & \multicolumn{3}{c}{ The cumulative error of torque $(\mathrm{N} / \mathrm{m})$} & \multicolumn{4}{c}{ The maximum distribution deviation of torque $(\mathrm{N} / \mathrm{m})$} \\
& $x$-axis & $y$-axis & $z$-axis & $x$-axis & $y$-axis & $z$-axis \\
\hline Pseudoinverse thruster allocation & 0.2568 & 0.1871 & 0.2222 & 0.0140 & 0.0102 & 0.0121 \\
Robust thruster allocation & 0.1185 & 0.0864 & 0.1901 & 0.0109 & 0.0079 & 0.0116
\end{tabular}




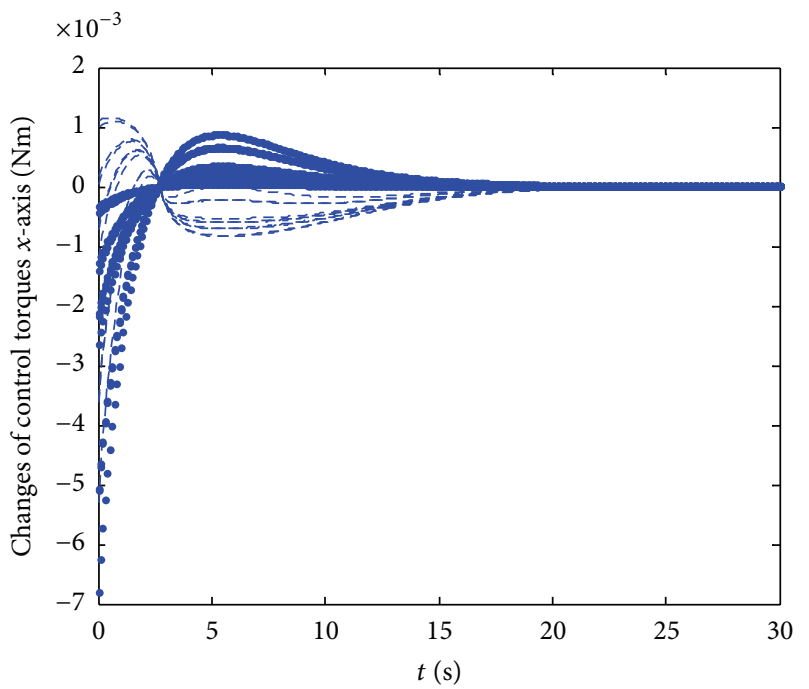

- Pseudoinverse allocation

- - Robust thruster allocation

(a)

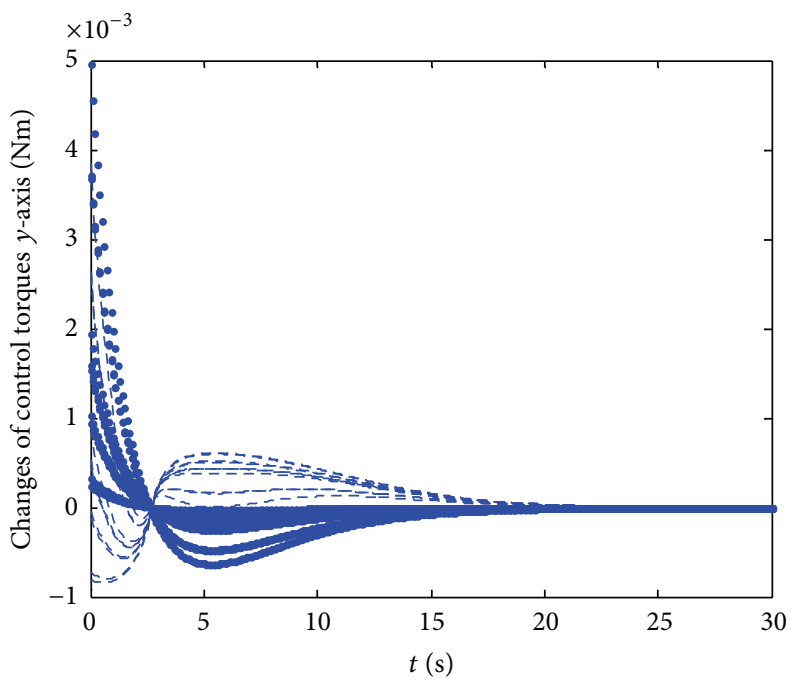

- Pseudoinverse allocation

- - Robust thruster allocation (Nm)

(b)

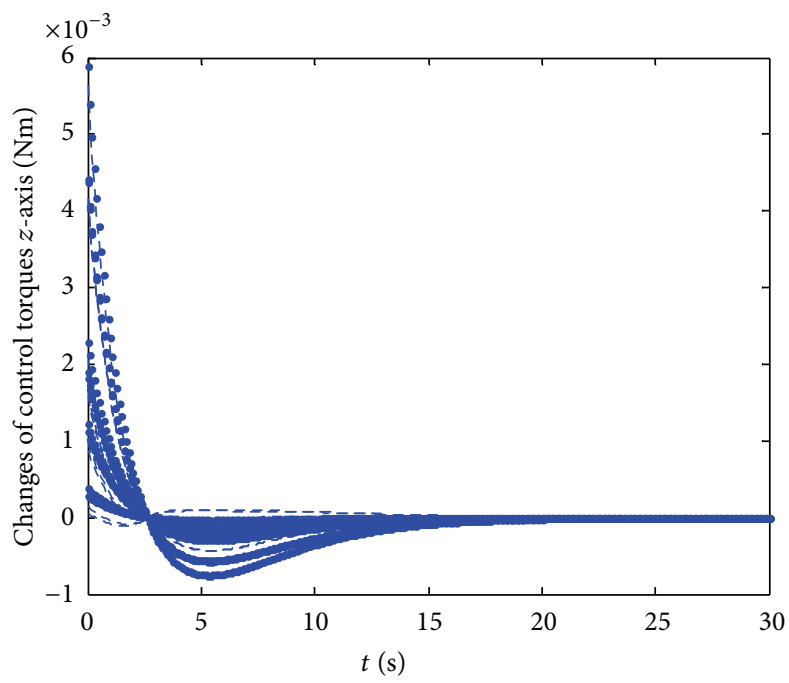

- Pseudoinverse allocation

- - - Robust thruster allocation

(c)

FIgURE 7: The curves of torque distribution deviation when the control efficiency matrix is with the uncertainty of $5 \%$.

TABLE 3: The simulation result when the control efficiency matrix is with the uncertainty of $5 \%$.

The cumulative error of torque $(\mathrm{N} / \mathrm{m}) \quad$ The maximum distribution deviation of torque $(\mathrm{N} / \mathrm{m})$

\begin{tabular}{lllllll} 
& $x$-axis & $y$-axis & $x$-axis & $y$-axis & $x$-axis & $y$-axis \\
\hline Pseudoinverse thruster allocation & 0.1077 & 0.0784 & 0.0931 & 0.0056 & 0.0041 & 0.0049 \\
Robust thruster allocation & 0.0583 & 0.0425 & 0.0765 & 0.0041 & 0.0030 & 0.0046
\end{tabular}


is transformed into a quadratic cone optimization problem and is solved by interior-point method. Finally, the feasibility and effectiveness of the robust thrust allocation algorithm is proved by three kinds of deviation. We can get the following conclusions:

(1) the uncertainty problem of control efficiency matrix can be effectively described using uncertainty ellipsoid set; at the same time, the model is more conducive to further derivation and simplification;

(2) by transforming the robust reference model to secondary cone optimization model, the problem of uncertainty model can be solved effectively, and the mathematical optimization method can be used conveniently for solving;

(3) compared with the traditional algorithm, multiple simulations show that the cumulative deviation of torque by using this robust thrust allocation algorithm decreases up to $20 \%$, and the maximum error of the distribution can be reduced more than $15 \%$, when the control efficiency matrix has uncertainty of $5 \%$ and $20 \%$. The control precision of thruster allocation link is improved.

\section{Conflict of Interests}

The authors declare that there is no conflict of interests regarding the publication of this paper.

\section{References}

[1] M. Bodson and S. A. Frost, "Control allocation with load balancing," in AIAA Guidance, Navigation, and Control Conference and Exhibit, August 2009.

[2] A. Bemporad, M. Morari, V. Dua, and E. N. Pistikopoulos, "The explicit linear quadratic regulator for constrained systems," Automatica, vol. 38, no. 1, pp. 3-20, 2002.

[3] P. Tøndel, T. A. Johansen, and A. Bemporad, "An algorithm for multi-parametric quadratic programming and explicit MPC solutions," Automatica. A Journal of IFAC, the International Federation of Automatic Control, vol. 39, no. 3, pp. 489-497, 2003.

[4] W. C. Durham, "Constrained control allocation," Journal of Guidance, Control, and Dynamics, vol. 16, no. 4, pp. 717-725, 1993.

[5] W. C. Durham, "Constrained control allocation: three-moment problem," Journal of Guidance, Control, and Dynamics, vol. 17, no. 2, pp. 330-336, 1994.

[6] J. D. Bošković, S.-M. Li, and R. K. Mehra, "Robust adaptive variable structure control of spacecraft under control input saturation," Journal of Guidance, Control, and Dynamics, vol. 24, no. 1, pp. 14-22, 2001.

[7] R. Zhou and J. Wang, "Adaptive control allocation between aerodynamic fin and side thruster and controller design using multi-objective optimization," Acta Aeronautica et Astronautica Sinica, vol. 28, no. 1, pp. 187-190, 2007.

[8] Y. Lu, Q. Hu, G. Ma, and J. Zhou, "Decentralized time-delay adaptive sliding mode control for attitude coordination of satellite formation under actuator misalignment," Acta Aeronautica et Astronautica Sinica, vol. 32, no. 9, pp. 1686-1695, 2011.
[9] Z. Li, M. Liu, H. R. Karimi, and X. Cao, "Observer-based stabilization of spacecraft rendezvous with variable sampling and sensor nonlinearity," Mathematical Problems in Engineering, vol. 2013, Article ID 902452, 11 pages, 2013.

[10] A. Zhang, Y. Wang, Z. Zhang, and H. R. Karimi, "Robust control allocation for spacecraft attitude stabilization under actuator faults and uncertainty," Mathematical Problems in Engineering, vol. 2014, Article ID 789327, 12 pages, 2014.

[11] D. Sheng, X. Yang, and H. R. Karimi, "Robust control for autonomous spacecraft evacuation with model uncertainty and upper bound of performance with constraints," Mathematical Problems in Engineering, vol. 2014, Article ID 589381, 16 pages, 2014.

[12] N. Wan, M. Liu, and H. R. Karimi, "Observer-based robust control for spacecraft rendezvous with thrust saturation," Abstract and Applied Analysis, vol. 2014, Article ID 710850, 10 pages, 2014.

[13] Y. Chen, X.-M. Dong, J.-P. Xue, and F.-W. Wang, "Robust predictive dynamic control allocation for uncertain actuators," Control Theory and Applications, vol. 29, no. 4, pp. 447-456, 2012.

[14] J.-J. Ma, Z.-Q. Zheng, and D.-W. Hu, "Subspace predictive dynamic control allocation for overactuated system with actuator dynamics," Acta Automatica Sinica, vol. 36, no. 1, pp. 130-138, 2010.

[15] K. A. Bordignon and W. C. Durham, "Null-space augmented solutions to constrained control allocation problems," in Proceedings of the AIAA Guidance, Navigation and Control Conference (AIAA-95-3209), Baltimore, Md, USA, 1995.

[16] C.-r. Sun and L. Huang, "Some extensions of robust linear optimization," Chinese Journal of Engineering Mathematics, vol. 24, no. 3, pp. 391-400, 2007.

[17] A. Ben-Tal and A. Nemirovski, "Robust solutions of linear programming problems contaminated with uncertain data," Mathematical Programming A, vol. 88, no. 3, pp. 411-424, 2000.

[18] J. A. M. Petersen and M. Bodson, "Interior-point algorithms for control allocation," in Proceedings of the AIAA Guidance, Navigation and Control Conference and Exhibit, pp. 1-43, Texas, Tex, USA, 2003. 


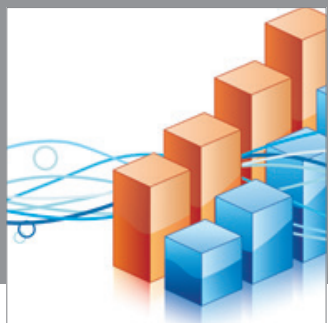

Advances in

Operations Research

mansans

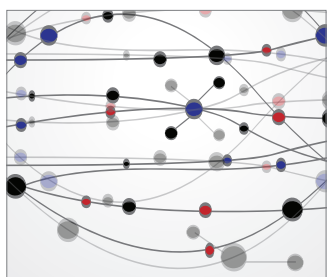

The Scientific World Journal
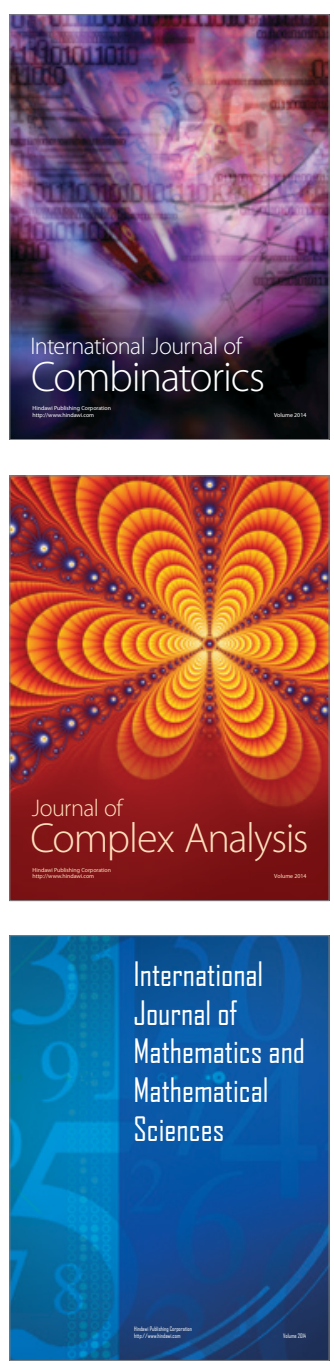
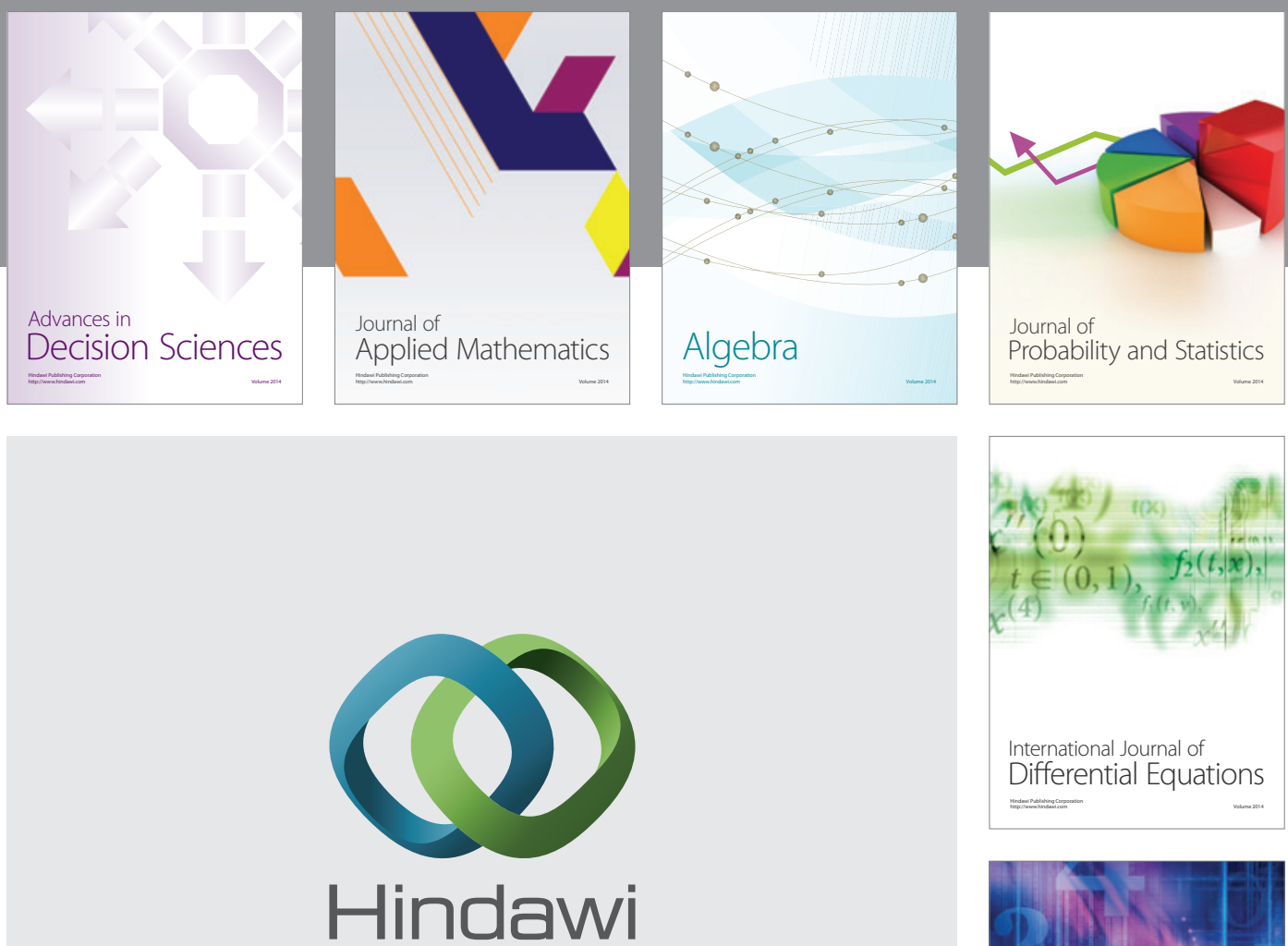

Submit your manuscripts at http://www.hindawi.com
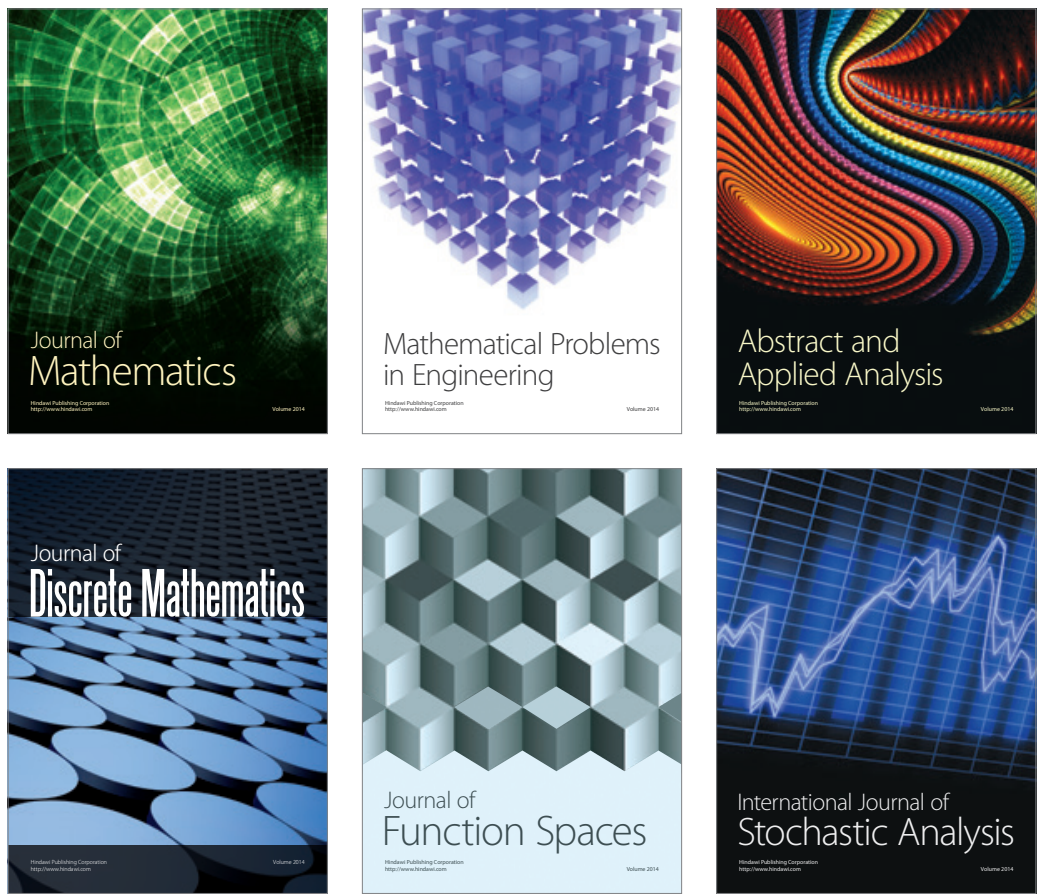

Journal of

Function Spaces

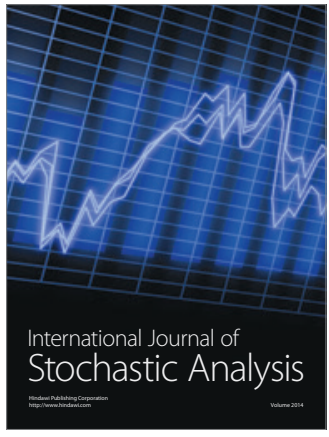

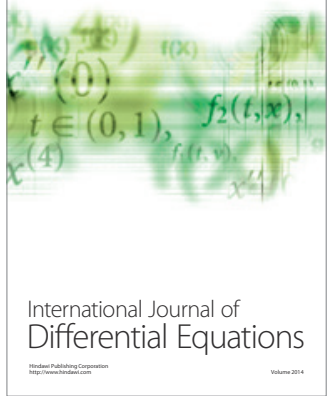
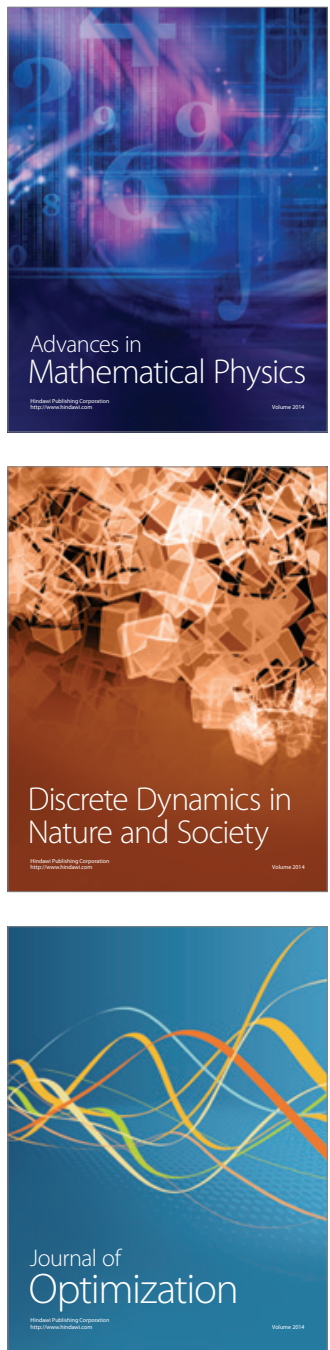